\title{
Effective étale-descent morphisms in the category of $M$-ordered sets
}

\author{
Pier Giorgio Basile
}

\begin{abstract}
A characterization of effective étale-descent morphisms in the category $M$-Ord of $M$-ordered sets, for a given monoid $M$, is obtained using the corresponding characterization in Cat of small categories.
\end{abstract}

Keywords. $M$-ordered sets - Descent data - Effective descent morphisms - Effective étale-descent morphisms - Discrete (op-)fibrations

\section{Introduction}

In [6] G. Janelidze and M. Sobral gave a complete characterization of the morphisms in the category Ord of (pre)ordered sets (denoted by Preord in [6]) which are effective for descent with respect to the class of étale morphisms, i.e., discrete fibrations. In [9] M. Sobral characterized the effective descent morphisms in the category Cat of small categories with respect to the class of discrete (op-)fibrations. These two works suggested the study of descent theory for the class of étale morphisms in the category $M$-Ord of $M$-ordered sets for a given monoid $M$. Using the identification of $M$-ordered sets as $M$-normed small categories given by M.M. Clementino, E. Colebunders and W. Tholen in [2], in this paper we present a complete characterization of the effective étale-descent morphisms in $M$-Ord.

\section{$2 \quad M$-ordered sets as $M$-normed categories}

Given a monoid $M$, consider the monad

$$
\mathbb{M}=(M \times(-), \mu, \eta)
$$

on Set, with $\mu_{X}: M \times M \times X \rightarrow M \times X$ defined by $(m, n, x) \mapsto(m n, x)$ and $\eta_{X}: X \rightarrow$ $M \times X$ by $x \mapsto(1, x)$, for each set $X$. The Barr extension [1] $\overline{\mathbb{M}}: \mathbf{R e l} \rightarrow \mathbf{R e l}$ is an extension of the monad $\mathbb{M}$, and it is given by

$$
(m, x)(\overline{\mathbb{M}} r)(n, y) \Longleftrightarrow m=n \quad \text { and } \quad x(r) y,
$$


where $r: X \rightarrow Y$ is a relation, $x \in X, y \in Y$, and $m, n \in M$. The category ( $\overline{\mathbb{M}}, 2)$-Cat of $(\overline{\mathbb{M}}, 2)$-categories and functors is the category $M$-Ord of $M$-ordered sets and equivariant maps. For a relation $a: M \times X \rightarrow X$ one writes $x \stackrel{m}{\rightarrow} y$ instead of $(m, x)(a) y$, that is $x$ is related to $y$ with weight $m$. As remarked in [2, Section V.1.4], this arrow notation for the structure of an $(\overline{\mathrm{M}}, 2)$-category $(X, a)$ emphasizes that $X$ is actually the object set of a small category, denoted again by $X$, with hom-sets

$$
X(x, y)=\{(x, m, y) \mid m \in M \quad \text { and } \quad x \stackrel{m}{\rightarrow} y\}
$$

for $x, y \in X$; moreover this small category comes equipped with a faithful functor

$$
\nu_{X}: X \rightarrow M, \quad(x, m, y) \mapsto m,
$$

with $M$ considered as a one-object category. Accordingly, the identity morphisms and composition in an $M$-ordered set $X$ are given by

$$
x \stackrel{e_{M}}{\rightarrow} x \quad \text { and } \quad(x \stackrel{m}{\rightarrow} y \quad \& \quad y \stackrel{n}{\rightarrow} z \Longrightarrow x \stackrel{n m}{\longrightarrow} z),
$$

while an equivariant map $f: X \rightarrow Y$ must satisfy

$$
x \stackrel{m}{\rightarrow} y \Longrightarrow f(x) \stackrel{m}{\longrightarrow} f(y)
$$

for all $x, y \in X$ and $m \in M$. Defining an $M$-norm to be a functor from the small category $X$ to the category $M$, we have a full embedding

$$
I:(\overline{\mathrm{M}}, 2) \text {-Cat } \hookrightarrow \text { Cat } \downarrow M, \quad(X, a) \mapsto\left(X, \nu_{X}\right) .
$$

Proposition 2.1 [2, Proposition V.1.4.2] The functor I is reflective and identifies $(\overline{\mathbb{M}}, 2)$ categories as those small categories over $M$ whose norm is faithful.

Now let $\mathbb{E}$ be the class of étale morphisms in $M$-Ord. As introduced in [3], a $(\mathbb{T}, 2)$ functor is étale if it is a pullback stable 'discrete fibration' (see [3] for details). For a cartesian monad, as $\mathbb{M}$ is, discrete fibrations are pullback stable, hence étale morphisms are the same as discrete fibrations. Using the arrow notation, an equivariant map $f$ : $X \rightarrow Y$ in $M$-Ord is an étale morphism if and only if:

$$
\forall x_{0} \in X, \quad \forall y_{1} \in Y, \quad \forall m \in M: y_{1} \stackrel{m}{\longrightarrow} f\left(x_{0}\right) \Longrightarrow \exists ! x_{1} \in f^{-1}\left(y_{1}\right): x_{1} \stackrel{m}{\longrightarrow} x_{0} .
$$

The problem concerning the characterization of effective étale-descent morphisms in $M$ Ord can be stated as follows.

Given an equivariant map $p: E \rightarrow B$ of $M$-ordered sets, denote by $\mathbb{E}(B)$ the slice category of étale morphisms over $B$ and by $p^{*}: \mathbb{E}(B) \rightarrow \mathbb{E}(E)$ the pullback functor along $p$. We have then a commutative (up to isomorphism) diagram

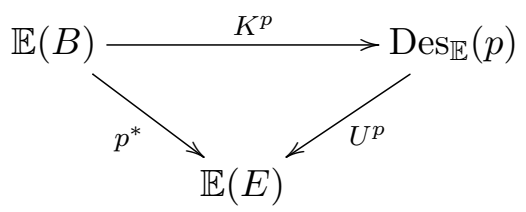


where $\operatorname{Des}_{\mathbb{E}}(p)$ is the category of $\mathbb{E}$-descent data for $p$, and $U^{p}$ and $K^{p}$ are the forgetful and the comparison functor, respectively. By definition, the equivariant map $p: E \rightarrow B$ is an (effective) étale-descent morphism if the comparison functor $K^{p}$ is full and faithful (an equivalence of categories). If we replace $\mathbb{E}$ by the class of all morphisms, $p$ is said to be an effective descent morphism. For a more detailed presentation of descent theory we refer the Reader to the papers [7] and [8].

\section{Characterization of (effective) étale-descent morphisms}

Consider the following diagram

$$
(\overline{\mathrm{M}}, 2)-\mathbf{C a t} \stackrel{I}{\rightarrow} \text { Cat } \downarrow M \stackrel{U}{\rightarrow} \text { Cat },
$$

where $I$ is the full embedding described in Section 2 and $U$ is the obvious forgetful functor. A functor $F: X \rightarrow Y$ in Cat is called a discrete fibration if for every object $x$ in $X$ and every morphism of the form $g: y^{\prime} \rightarrow F(x)$ in $Y$ there exists a unique morphism $f: x^{\prime} \rightarrow x$ in $X$ such that $F(f)=g$. The notion of 'discrete fibration' given in Section 2 for a morphism in $M$-Ord coincides with the notion of discrete fibration given above when we consider $M$-ordered sets as ( $M$-normed) small categories. Moreover, given an equivariant map $p: E \rightarrow B$ of $M$-ordered sets, since $I$ preserves pullbacks, the pullback functor $p^{*}: \mathbb{E}(B) \rightarrow \mathbb{E}(E)$, where $\mathbb{E}$ is the class of étale morphisms in $M$-Ord, is described by the following diagram

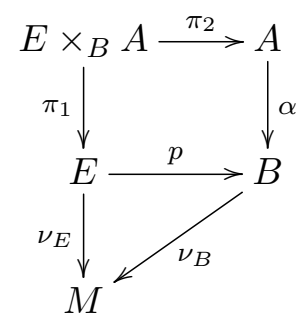

where the square is in Cat. In fact for a discrete fibration $\alpha: A \rightarrow B$ of small categories, being in particular a faithful functor, the composition

$$
A \stackrel{\alpha}{\rightarrow} B \stackrel{\nu_{B}}{\longrightarrow} M
$$

gives an $M$-valued norm for $A$ making $\alpha: A \rightarrow B$ an object in $\mathbb{E}(B)$. Hence the arguments given in [9] leading to the characterization of effective descent morphisms in Cat with respect to the class of discrete (op-)fibrations can be used to get a characterization of the effective étale-descent morphisms in $M$-Ord.

Following those arguments, the equivariant map $p: E \rightarrow B$ can be then factorized in 
Cat in the following way

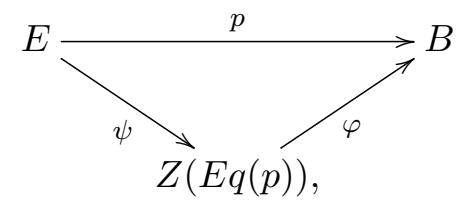

where $Z(E q(p))$ is the category of zigzags with $E$ as object-set and morphisms given by equivalent classes of zigzags of the form

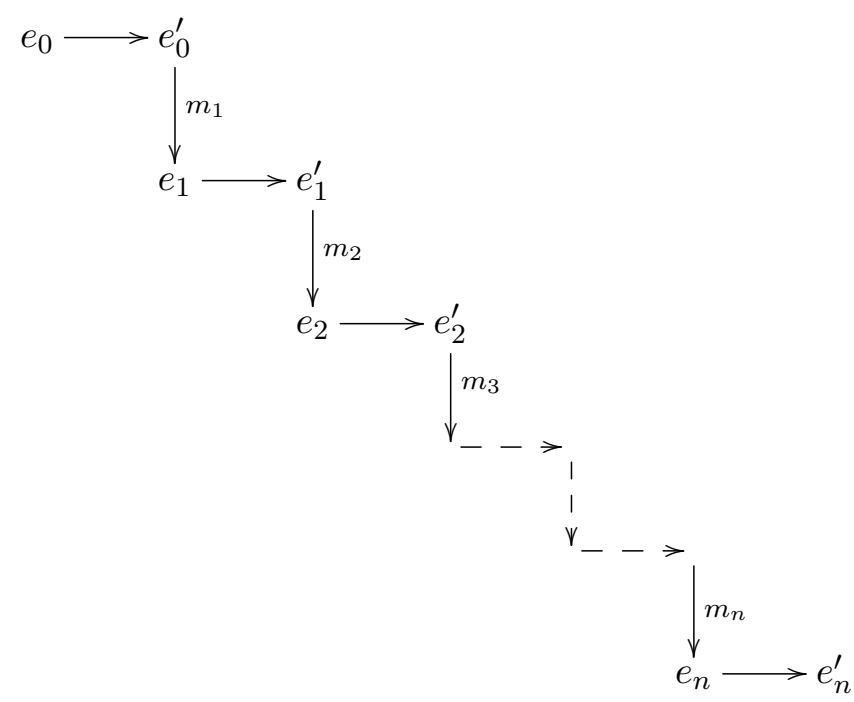

where $p\left(e_{i}\right)=p\left(e_{i}^{\prime}\right)$ for $i=0, \cdots, n$, and $e_{i}^{\prime} \stackrel{m_{i+1}}{\longrightarrow} e_{i+1}$ in $E$ for $i=0, \cdots, n-1$. The equivalent classes are given by the smallest equivalence relation $\sim$ for which

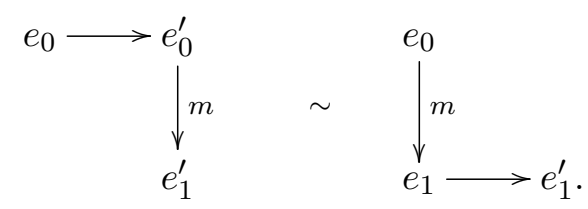

For a more detailed presentation of this construction we refer the Reader to the papers [6] and [9]. An $n$-zigzag as above will be denoted by

$$
\left[e_{n}, e_{n}^{\prime}\right] m_{n} \cdots m_{2}\left[e_{1}, e_{1}^{\prime}\right] m_{1}\left[e_{0}, e_{0}^{\prime}\right]
$$

The morphism $\psi$ is defined as the identity on objects and $\psi\left(e_{0} \stackrel{m}{\rightarrow} e_{1}\right)=\left[e_{0} \stackrel{m}{\rightarrow} e_{1}\right]$ on morphisms, while $\varphi$ on objects acts as $p$ and the image of an equivalent class of an $n$-zigzag $\left[e_{n}, e_{n}^{\prime}\right] m_{n} \cdots m_{2}\left[e_{1}, e_{1}^{\prime}\right] m_{1}\left[e_{0}, e_{0}^{\prime}\right]$ via $\varphi$ is

$$
p\left(e_{0}\right) \stackrel{m_{1}}{\longrightarrow} p\left(e_{1}^{\prime}\right) \stackrel{m_{2}}{\longrightarrow} \cdots \stackrel{m_{n}}{\longrightarrow} p\left(e_{n}^{\prime}\right)=p\left(e_{0}\right) \stackrel{m_{n} \cdots m_{2} m_{1}}{\longrightarrow} p\left(e_{n}^{\prime}\right) .
$$


Based on the observations above, the Theorem below immediately follows form the similarly formulated [9, Theorem 2], while the Corollary can be deduced either directly from it, or, easily, from [9, Corollary 3].

Theorem 3.1 An equivariant map $p: E \rightarrow B$ is an effective étale-descent morphism in $M$-Ord if and only if $\varphi: Z(E q(p)) \rightarrow B$ is a full and faithful lax epimorphism in Cat.

Corollary 3.2 An equivariant map $p: E \rightarrow B$ is an effective étale-descent morphism in $M$-Ord if and only if

(i) For each $p(e) \stackrel{k}{\rightarrow} p\left(e^{\prime}\right)$ in $B$ with $k \in M$ there exists a zigzag in $Z(E q(p))$

$$
\left[e_{n}, e_{n}^{\prime}\right] m_{n} \cdots m_{2}\left[e_{1}, e_{1}^{\prime}\right] m_{1}\left[e_{0}, e_{0}^{\prime}\right]
$$

with $k=m_{n} \ldots m_{2} m_{1}$, and such a zigzag is unique up to equivalence.

(ii) every point $b \in B$ is in relation to a point of the image via a right-invertible element of the monoid, i.e., for each $b \in B$ there exist $e \in E, n, m \in M$ such that $p(e) \stackrel{n}{\rightarrow} b$ and $b \stackrel{m}{\rightarrow} p(e)$ with $n m=1$.

Remarks 3.3 1. When $M=1,1$-Ord=Ord, also identified as the full subcategory of Cat given by small categories $X$ for which $X \rightarrow 1$ is faithful. Hence the characterization of effective étale-descent morphsism in $M$-Ord generalizes the characterization in Ord given in [6]. As in Ord uniqueness of zigzags encodes the fact that $Z(E q(p))$ is a (pre)order, here it encodes the property that $Z(E q(p))$ is an $M$-ordered set, with norm $Z(E q(p)) \stackrel{\varphi}{\rightarrow} B \stackrel{\nu_{B}}{\longrightarrow} M$.

2. The étale-descent morphisms in $M$-Ord are precisely the morphisms for which condition (ii) is satisfied.

3. Since $\varphi^{\mathrm{op}}$ is a full and faithful lax epimorphism if and only if the same holds for $\varphi$, we conclude, as in the case for Cat, that the effective descent morphisms in $M$ Ord with respect to the class of discrete op-fibrations coincide with the effective étale-descent morphisms.

4. Effective descent morphisms in $M$-Ord were characterized in [4, Theorem 1.8] as the equivariant maps $p: E \rightarrow B$ such that

$$
\forall \quad b_{2} \stackrel{m}{\rightarrow} b_{1} \stackrel{n}{\rightarrow} b_{0} \quad \text { in } B \quad \exists \quad e_{2} \stackrel{m}{\rightarrow} e_{1} \stackrel{n}{\rightarrow} e_{0} \quad \text { in } E: \forall i=0,1,2 \quad p\left(e_{i}\right)=b_{i} .
$$

Therefore every effective descent morphism is effective for étale-descent. The converse is not true, even for surjective maps, as illustrated by the following modification of [5, Example 8.7]: 


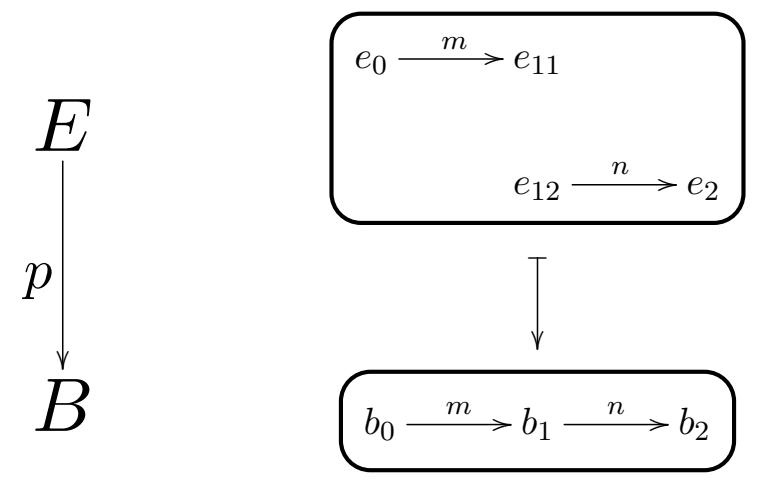

\section{Acknowledgements}

Research supported by the Centro de Matemática da Universidade de Coimbra (CMUC), funded by the European Regional Development Fund through the program COMPETE and by the Portuguese Government through the FCT-Fundação para a Ciência e a Tecnologia under the project PEst-C/MAT/UI0324/2013 and grant number $\mathrm{SFRH} / \mathrm{BD} / 85837 / 2012$. This work has been realized during my $\mathrm{PhD}$ program. I wish to thank my supervisor Maria Manuel Clementino for her precious help and support.

\section{References}

[1] M. Barr, Relational Algebras, in: Reports of the Midwest Category Seminar, IV, pp 39-55, Lecture Notes in Mathematics 137, Springer, Berlin. (1970).

[2] M.M. Clementino, E. Colebunders and W. Tholen, Lax algebras as spaces, in: Monoidal Topology, pp 375-466, Encyclopedia Math. Appl. 153, Cambridge Univ. Press, Cambridge (2014).

[3] M.M. Clementino, D. Hofmann and G. Janelidze, On exponentiability of étale algebraic homomorphisms, J. Pure Appl. Algebra 217 (2013), 1195-1207.

[4] M.M. Clementino, D. Hofmann and A. Montoli, Covering Morphisms in Categories of Relational Algebras, Appl. Categ. Structures, 22 (2014), 767-788.

[5] G. Janelidze and M. Sobral, Finite preorders and topological descent I, J. Pure Appl. Algebra 175 (2002), 187-205.

[6] G. Janelidze and M. Sobral, Finite preorders and topological descent II: étale descent, J. Pure Appl. Algebra 174 (2002), 303-309.

[7] G. Janelidze and W. Tholen, Facets of Descent I, Appl. Categ. Structures 2 (1994), 1-37.

[8] G. Janelidze and W. Tholen, Facets of Descent II, Appl. Categ. Structures 5 (1997), 229-248. 
[9] M. Sobral, Descent for Discrete (Co)fibrations, Appl. Categ. Structures 12 (2004), 527-535. 